\title{
Catalogue of variable stars in Milky Way globular clusters
}

\author{
http://www . astro. utoronto.ca/ cclement/read.html \\ Christine Clement ${ }^{1, \star}$ \\ ${ }^{1}$ Department of Astronomy \& Astrophysics, University of Toronto, 50 St. George St., Toronto, ON, M5S 3H4, \\ Canada
}

\begin{abstract}
Globular cluster variable stars have been studied for more than a century. In the early investigations, more than $90 \%$ of the known variables were of the RR Lyrae type. However, in the interim, technological advances have facilitated the discovery of other types of variables. As a result, although RR Lyrae stars still dominate, they now constitute less than $70 \%$ of the known variables.
\end{abstract}

\section{Introduction}

A summary paper based on this catalogue was published in the Astronomical Journal by Clement et al. (2001) [1]. Since 2001, the number of known (optical/near infrared) variables has almost doubled because numerous investigations have dealt with variable stars in globular clusters. Combining the new results with summaries published by Helen Sawyer Hogg in 1939, 1955, and 1973 ([2-4]) illustrates how our knowledge of the variable star content of Galactic globular clusters has changed over the past eight decades.

\section{Globular cluster variables: 1939-2016}

Observations of globular cluster variables in the early 20th century were usually made with photographic plates. This practice continued until the 1980s. Only the brightest variables could be observed and variations of less than $\sim 0.3$ mag were not detectable. Consequently, more than $90 \%$ of the known variables were of the RR Lyrae type. The rest were Cepheids and long period variables, which were among the brightest stars in clusters. Variables below the horizontal branch in the colour-magnitude (CM) diagram were too faint to be discovered.

The routine use of CCD detectors since the 1980s has changed that. Because CCDs are more efficient than photographic detectors, it is possible to observe fainter stars and to detect smaller variations. Also, since exposure times can be shorter, it is feasible to detect eclipses and other brightness changes on time scales of half an hour or less.

As a result of these developments, numerous SX Phe stars and binaries have been identified. They now constitute more than $15 \%$ of the known variables. The number of slow variables has also increased because many stars near the red giant tip in the CM diagram exhibit low level variations.

^cclement@astro.utoronto.ca 
In future, the percentage of known binaries will undoubtedly increase because advances in image processing techniques and the use of space telescopes make it possible to study stars in crowded central regions where most binaries are located.

Table 1. Census of globular cluster variable stars.

\begin{tabular}{lccccr}
\hline Variables & 1939 & 1955 & 1973 & 2001 & 2016 \\
\hline RR Lyrae & 613 & 1114 & 1202 & 1842 & $>2925$ \\
Type II Cep, RV Tau & 27 & 32 & 37 & 60 & $>85$ \\
Slow Vars: L, M, SR & 15 & 53 & 71 & 117 & $>475$ \\
SX Phe & 0 & 0 & 0 & 117 & $>325$ \\
Eclipsing, ELL & 1 & 2 & 2 & 96 & $>400$ \\
\hline
\end{tabular}

The above table lists the number of pulsating and binary variables catalogued at five different epochs. It includes only the stars that were assumed to be cluster members, but membership status can often be ambiguous. In the early days, it was based on apparent magnitude and distance from the cluster centre. Then in the second half of the twentieth century, CM diagrams became available for many clusters so that the magnitude and colour were used. Also, in a few cases, mainly for the brightest stars, radial velocities and proper motions could be derived so that the cluster members could be distinguished from stars in the surrounding field. Now, with improved astrometry, it is becoming routine to derive proper motions for cluster stars.

Acknowledgments: I am pleased to acknowledge the support that I have received from Peter Martin, Howard Yee, and Ray Carlberg, the three people who have served as chairs of the University of Toronto Astronomy \& Astrophysics department since my retirement in 2006. They have all helped me in my research by giving me access to departmental resources. I am also grateful to the worldwide astronomers who have cited the 2001 paper and encouraged me to continue with this project. Finally, I would like to thank Márcio Catelan who inspired me to publish the 2001 paper.

\section{References}

[1] Clement, C. M., Muzzin, A., Dufton, Q., Ponnampalam, T., Wang, J., Burford, J., Richardson, A., Rosebery, T., AJ, 122, 2587 (2001)

[2] Sawyer, H. B., David Dunlap Publ., 1, No. 4 (1939)

[3] Sawyer, H. B., David Dunlap Publ., 2, No. 2 (1955)

[4] Sawyer Hogg, H., David Dunlap Publ., 3, No. 6 (1973) 